\title{
Dr. Lore Maria Peschel-Gutzeit, 1. Vorsitzende des djb (1977-1981) und Ehrenpräsidentin des Verbands feiert ihren 80. Geburtstag
}

\section{Dr. Lore Maria Peschel-Gutzeit - Vita}

Lore Maria Peschel-Gutzeit, geboren am 26. Oktober 1932 in Hamburg, studierte von 1951 bis 1955 Rechtswissenschaft in Hamburg und Freiburg im Breisgau. 1955 absolvierte sie mit 22 Jahren ihr Erstes Staatsexamen, 1959 folgte nach dem Referendariat in Hamburg, München, Speyer und Freiburg i.Br. das Zweite Staatsexamen. Zunächst war sie - von 1959 bis 1960 - Rechtsanwältin in Freiburg. Anschließend begann sie ihre Karriere als Richterin: bis 1972 am Landgericht Hamburg, danach bis 1991 am Hanseatischen OLG ebenfalls in Hamburg. 1978 wurde sie dort Familienrichterin und 1984 erste Präsidentin eines Familiensenates am Hanseatischen Oberlandesgericht.

Mitglied der SPD ist sie seit 1988. Von 1991 bis 1993 war sie Senatorin für Justiz in Hamburg, von 1994 bis 1997 Senatorin für Justiz in Berlin. Von 1995 bis 1996 war sie auch Mitglied des Abgeordnetenhauses von Berlin, Bezirksliste Tempelhof. Von 1997 bis 2001 war sie ein weiteres Mal Senatorin für Justiz in Hamburg. 2002 erfolgte ihre erneute Zulassung als Rechtsanwältin. Heute ist sie in der Berliner Kanzlei Kärgel de Maizière \& Partner tätig.

1990 promovierte sie zum Thema „Das Recht zum Umgang mit dem eigenen Kinde“. Als Mitglied der Verfassungskommission wirkte sie an dem seit 1994 gültigen Gesetzeszusatz mit, der die staatliche Förderung der Gleichberechtigung von Frauen und Männern festschrieb.

Am 7. Juli 2004 verlieh ihr der Regierende Bürgermeister der Stadt Berlin Klaus Wowereit die Stadtältestenwürde. Am 4. Oktober 2004 folgte die Verleihung des Verdienstordens der Bundesrepublik Deutschland 1. Klasse durch Bundespräsident Johannes Rau im Rahmen eines Festaktes im Schloss Charlottenburg. Sie war und ist Mitglied zahlreicher Vereinigungen, u.a. ver.di (früher ÖTV), Schadow-Gesellschaft Berlin,
Deutscher Anwaltverein, Deutscher Juristentag, Deutscher Familiengerichtstag, Kuratorium Deutsche Liga für das Kind, Deutscher Kinderschutzbund, Landesfrauenrat Hamburg (Vorsitzende 1990 von 1992), Übersee-Club Hamburg, Capital Club Hamburg, International Club.

Außerdem ist Lore Maria Peschel-Gutzeit seit den Fünfzigerjahren Mitglied des djb.

Sie war in vielen Funktionen aktiv: Als Ersatzmitglied des Bundesvorstands (1967-1969) setzte sie das „Lex Peschel“ durch, das Beamtinnen und Richterinnen Teilzeiturlaub und Familienurlaub gewährt. Sie war Vorsitzende der Kommission Teilzeitarbeit von Beamtinnen (1968), Mitglied des erweiterten Bundesvorstands (1973-1975), Vorsitzende der großen Familienrechtskommission zu den noch offenen Fragen der Familienrechtsreform (1975), 2. Vorsitzende des djb (1975-1977), Vorsitzende der Fachkommission Familienrecht: elterliche Sorge (1976) und Vorsitzende der Kommission zur Neuordnung des Rentenrechts (1976). Dann wurde sie zur 1. Vorsitzenden des djb (1977-1981) gewählt. Anschließend war sie wiederum in den Kommissionen tätig: als Vorsitzende der Familienrechtskommission zur geplanten Änderung des Scheidungs- und Scheidungsfolgenrechts (1983), Vorsitzende der Kommission Jugendhilfe (1984-1989), Vorsitzende der Familienrechtskommission zum Entwurf des Unterhaltsänderungsgesetzes und zum Entwurf eines Gesetzes über weitere Maßnahmen auf dem Gebiet des Versorgungsausgleichs (1985), Vorsitzende der Familienrechtskommission (19881996), Leiterin der Unterkommission Unterhaltsrecht (19901992) und Vorsitzende der Kommission Anerkennungskultur (2004-2005). 2006 erhielt sie die Ehrennadel des djb für über 50-jährige Verbandsmitgliedschaft. 2008 wurde sie anlässlich des 6o. Verbandsgeburtstags zur Ehrenpräsidentin des Deutschen Juristinnenbunds ernannt.

\section{Die Kolleginnen gratulieren}

Renate Damm, Rechtsanwältin, 1. Vorsitzende von 1983 bis 1989 , Ehrenpräsidentin:

Dr. Lore Maria Peschel-Gutzeit ist die „geborene“ 1. Vorsitzende des djb! So habe ich sie in Anlehnung an die „geborenen Orderpapiere“ des Handelsrechts gerne bezeichnet („tralalakaukauboko“ lautet die studentische Merkformel). Ihre Tätigkeit als 1. Vorsitzende hatte „Gesetzeskraft“! Das musste auch der Gesetzgeber bei der Regelung des Scheidungsfolgenrechtes 1985 erfahren. Lore Maria Peschel-Gutzeit als Vorsitzende der Familienrechtskommission des djb und ich als amtieren- de 1. Vorsitzende setzten in einer Nacht- und Nebelaktion die Forderungen des djb durch. Das letzte Telefonat mit dem damaligen Justizminister Hans Arnold Engelhard fand nachts um 4 Uhr statt. Es war eine großartige konzertierte Aktion mit den Abgeordnetinnen aller Bundestagsfraktionen!

Wenn es um die Sache ging, kannte Dr. Lore Maria PeschelGutzeit kein „Pardon“. Da schreckte sie auch nicht vor verbotener Kinderarbeit zurück! In mühevoller Kleinarbeit fertigte sie mit ihren Kindern die Stimmzettel für die Mitgliederversammlung an. 
Gerne erinnere ich mich auch an ihr strenges Regiment, wenn es darum ging, für einen gemeinsamen Auftritt beim Bundesverfassungsgericht die vorgegebene Redezeit nachts im Hotel zu proben. Sie hielt natürlich genau ihre Zeit ein. Der damalige Präsident des Bundesverfassungsgerichts und Vorsitzende des 1. Senats, Professor Roman Herzog, forderte sie jedoch auf fortzufahren. Es bedarf keiner weiteren Erklärung, dass mit der von Dr. Lore Maria Peschel-Gutzeit gegebenen Begründung der Verfassungsbeschwerde stattgegeben wurde.

Antje Sedemund-Treiber, Präsidentin des Bundespatentgerichts i.R., 1. Vorsitzende von 1989 bis 1993, Ehrenpräsidentin:

Lore Maria Peschel-Gutzeit war meine erste Begegnung mit dem djb in persona: Bei einem Vortrag über Neuerungen des Ersten Eherechtsreformgesetzes befand sie sich unter meinen Zuhörerinnen. Nach dem Vortrag kam sie auf mich zu und fragte ohne Umschweife, ob ich nicht Mitglied des djb werden wollte. Natürlich hatte ich von diesem Verband gehört und auch schon vorsichtig über eine Mitgliedschaft nachgedacht. Immer aber fehlte die Zeit, mich näher damit zu beschäftigen. Lore Maria Peschel-Gutzeit überbrückte meine Bedenken energisch und so wurde ich stante pede Mitglied unseres Verbandes.

Bei dieser Begegnung mit Lore Maria Peschel-Gutzeit wusste ich nicht, wie viel sie und der djb bereits für mich getan hatten. Öffentlich Bedienstete wie seinerzeit ich als Beamtin im Bundesministerium der Justiz hatten ihrem Dienstherren ursprünglich ihre volle Arbeitskraft

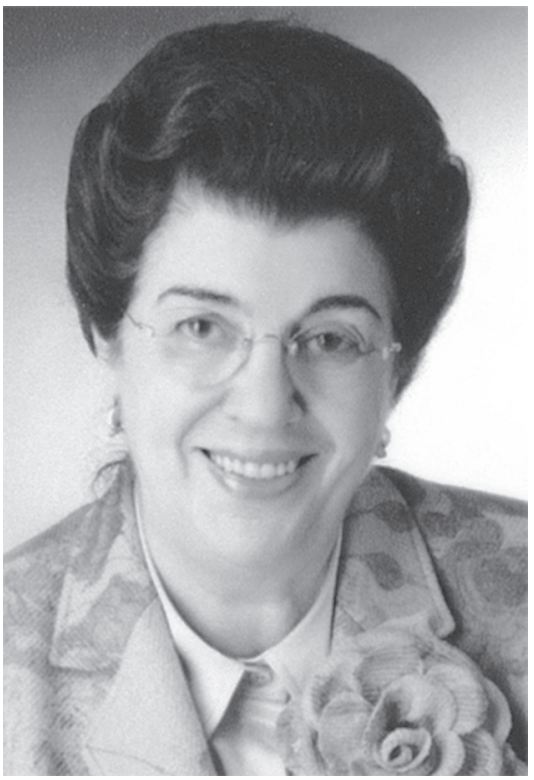

D Dr. Lore Maria Peschel-Gutzeit funden werden, eine Aufgabe, deren Lösung sich hinzog. Lore Maria Peschel-Gutzeit hatte die Kommission mit enormem Einsatz, großer Kompetenz und ebenso viel Erfahrung geleitet und sowohl im Bundestag als auch beim Bundesverfassungsgericht beachtliche Erfolge für die Rechte von Frauen erstritten. Da lag die Latte für jede Nachfolgerin hoch, zumal die Senatorin der Kommission erhalten blieb und die Arbeit weiter begleitete. Die höchste Form eines kritischen Kommentars bestand - neben dem Sachargument - darin, dass sie mitten in der Diskussion vom „Du“ zum „Sie“ wechselte.

In den zehn Jahren als Senatorin für Justiz in Hamburg, Berlin und dann wieder in Hamburg hat sie unserer Justitia konsequent und nachhaltig kompetente Juristinnen zugeführt.

Ihre berührende Rede am Grabe der langjährigen Freundin und Weggefährtin Adelheid Koritz-Dohrmann ließ alle Anwesenden die tiefe Verbundenheit dieser beiden kämpferischen, tapferen und lebensklugen Frauen spüren.

Seit zehn Jahren lebt Lore Maria in Berlin und so begegnen wir uns erfreulicherweise immer wieder. Mit nach wie vor hamburgischem Understatement bringt sie ihre Lebenserfahrung schnell auf den Punkt. „Wer rausgeht, muss ja wieder reinkommen “ gehört dazu. Und ihr immer wieder zu hörendes „Mir sacht ja keiner was“ lässt mich hoffen, dass sie weiter präsent sein wird. Möge sie uns noch lange erhalten bleiben.

Prof. Dr. Ursula Nelles, Hochschulrektorin, 1. Vorsitzende von 1997 bis 2001:

Geschichten aus drei Rathäusern

Auch wenn man sich bei Lore Maria Peschel-Gutzeit in dieser Frage nicht sizur Verfügung zu stellen; Teilzeitarbeit oder andere Erleichterungen gab es nicht. Eine von Lore Maria Peschel-Gutzeit mit dem djb initiierte Gesetzesnovelle änderte dies. Zukünftig konnten im öffentlichen Dienst Beschäftigte ihre Tätigkeit befristet aussetzen oder auf Teilzeit beschränken, um Kinder oder pflegebedürftige Angehörige zu betreuen. Wegen meiner Söhne hatte ich hiervon Gebrauch gemacht. Mit Teilzeitarbeit habe ich die frühen Jahre der Kinderbetreuung meistern können und vor allem den Anschluss an meinen Beruf behalten, den aufzugeben mir sehr schwer geworden wäre. Als mich Lore Maria Peschel-Gutzeit auf eine Mitgliedschaft im djb ansprach, war mir nicht bekannt, dass sie und der djb mit der sogenannten „Lex Peschel-Gutzeit“ mit diese Chance eröffnet hatten: Danke, Lore Maria, danke djb!

Ursula Raue, Rechtsanwältin, 1. Vorsitzende von 1993 bis 1997:

Die Familienrechtskommission des djb, das war über viele Jahre Dr. Lore Maria Peschel-Gutzeit. Als sie 1991 Senatorin für Justiz in Hamburg wurde, musste eine Nachfolgerin ge- cher sein kann: Ein 80. Geburtstag sollte kein Arbeitstag sein! Deshalb möchte ich Anekdoten beisteuern, die für mich untrennbar mit Lore Maria Peschel-Gutzeit verbunden sind, sich aber ausschließlich bei festlichen Ereignissen des djb zugetragen haben:

Zum Empfang der 29. Arbeitstagung (1991) in Lübeck erschien der Bürgermeister verspätet und reichlich „indisponiert“. Als er lallend verkündete, er möge keine Frauen in der Politik, schon gar nicht in Lübeck, mochten die etwa 300 Teilnehmerinnen zunächst nicht glauben, was da geschah. Die Ehrenpräsidentin des djb, Lore Maria Peschel-Gutzeit, zog hingegen sofortige Konsequenzen: wortlos aber mit körpersprachlich präziser Ansage marschierte sie aus dem Saal - und alle folgten ihr.

Das 50-jährige Bestehen des djb (1998) wurde in Dortmund gefeiert. Insgesamt war die Tagung vergnüglich, aber die Eröffnung im Rathaus lief zeitlich völlig aus dem Ruder. Noch während des Empfangs erhielt ich als damalige Vorsitzende von der Ehrenpräsidentin klare Hinweise zum Qualitätsma- 
nagement. Sie wirken bis heute: Kein von mir verantworteter Festakt hat seitdem länger als 90 Minuten gedauert.

Den 34. Kongress (2001) durfte der djb im Kaisersaal des Hamburger Rathauses eröffnen. Lore Maria Peschel-Gutzeit war Justizsenatorin und fungierte als Hausherrin. Ich dachte: Das ist genau der richtige Rahmen für diese große und starke Frau!

Hoch soll sie leben!!

Margret Diwell, Rechtsanwältin, Präsidentin des Berliner Verfassungsgerichtshofs (2007-2012), Präsidentin von 2001 bis 2005: Liebe Lore Maria, Dein Wort hat Gewicht!

Ich wünsche Dir und uns, dass Du auch in Deinem kommenden Lebensjahr die Stimme laut und deutlich erhebst. Nein, ich meine nicht die Fortbildung für Rechtsanwältinnen und Richter. Ich denke eher an Themen, die Dich seit Jahrzehnten begleiten und doch noch immer nicht erledigt sind, weil der Fortschritt eben eine Schnecke und die Gleichberechtigung und Gleichstellung der Frau in Gesellschaft, Beruf und Familie wie die rechtliche Absicherung der Lebenssituation von Kindern und alten Menschen eine Aufgabe für Generationen ist. So hast Du vor 31 (!) Jahren als 1. Vorsitzende des djb die Abschaffung des Ehegattensplittings gefordert. Heute droht stattdessen das Betreuungsgeld. Die UN-Kinderrechtskonvention von 1989 ist nicht umgesetzt. Die Kinderrechte stehen nicht im Grundgesetz. Dafür müssen Kinder im Elternstreit einer Vielzahl von fremden Personen Rede und Antwort stehen („Wen hast du lieber: Papa oder Mama und wenn ja warum?“), deren Qualifikation niemand überprüft. Und der djb hat noch immer keine Traditionsbeauftragte, Dein Vorschlag von 2004, der in unserer rasenden Zeit neu bedacht werden sollte. Die richtig fiesen Kindschaftssachen aber und Richter, die nach eigener monatelanger Untätigkeit die Eskalation des Konflikts bei den Anwältinnen verorten, würde ich Dir im nächsten Jahr gerne ersparen. Gesundheit und Glück wünsche ich Dir für Dein kommendes Lebensjahr und vielleicht darf es doch ein wenig öfter und länger ein schönes Hotel z.B. auf Rügen sein?

Jutta Wagner, Rechtsanwältin und Notarin, Präsidentin von 2005 bis 2011:

Lore Maria Peschel-Gutzeit habe ich für Vieles zu danken.

Schon als ich sie und den kämpferischen djb noch gar nicht kannte, waren sie und ihre politischen Aktionen wichtig für die Art von Leben, für das ich mich als 1949 geborene junge Frau entscheiden konnte, ein gleichberechtigtes Leben ohne gesetzlich festgeschriebene Männerdominanz, mit einem Gleichberechtigungsgesetz seit 1. Januar 1958, der Familienrechtsreform von 1977, der Reform des $\mathbb{S} 218$ StGB und einem Artikel 3 GG, der endlich einen Handlungsauftrag für den Gesetzgeber enthält. Für all dieses, mir und den Jüngeren Selbstverständlichkeiten, musste Lore Maria Peschel-Gutzeit mit ihren Verbündeten heftige Schlachten schlagen. Ich wusste nichts von ihr, kannte nicht einmal ihren Namen, wusste auch nicht, wieviel Mut und Einsatz nötig gewesen waren.
Lore Maria Peschel-Gutzeits Name wurde mir dann selbstverständlich geläufig, als ich Anfang der 80er Jahre dem djb beitrat. Als Mensch von Fleisch und Blut in meinem Leben präsent ist Lore Maria Peschel-Gutzeit jedoch erst, seit sie 2002 ihre Tätigkeit als Anwältin in Berlin aufgenommen hat. Seitdem darf ich mich über viele, viele kollegiale und freundschaftliche Begegnungen freuen. In meinen sechs Jahren als djb-Präsidentin gehörte sie zu denjenigen, auf deren politische und auch ganz praktische Unterstützung ich wirklich zählen konnte - keine Selbstverständlichkeit. Besonders berührend und von herausragender Einmaligkeit in meinem Leben war sicherlich der Augenblick, als es mir qua Präsidentinnenamt beim 60. Geburtstag des djb zufiel, Lore Maria Peschel-Gutzeit, Renate Damm und Antje Sedemund-Treiber im Großen Protokollsaal des Reichstagsgebäudes zu Ehrenpräsidentinnen des djb ernennen zu dürfen. Das Stichwort Anerkennungskultur, von Lore Maria Peschel-Gutzeit in die djb-Vorstandsdiskussion eingebracht, wird sicher nicht vergessen werden.

Past President wäre ich übrigens nicht, wenn nicht diese Funktion auf Vorschlag von Lore Maria Peschel-Gutzeit in die Satzung des djb aufgenommen worden wäre. Ich freue mich auf viele weitere gemeinsame Aktionen und auch das eine oder andere gemeinsame Glas Wein - nur wer das Leben zu genießen versteht, und das tut Lore Maria Peschel-Gutzeit mit Sicherheit, hat auf Dauer die Kraft zu kämpfen!

\section{Ramona Pisal, Vors. Richterin am OLG, Präsidentin seit 2011:}

Vor beinahe 20 Jahren habe ich sie zum ersten Mal erlebt, die damalige Senatorin für Justiz Dr. Lore Maria Peschel-Gutzeit. Auf dem Deutschen Jugendgerichtstag in Potsdam sprach sie über Jugendkriminalität, offen, ohne die Tatsachen zu beschönigen, und doch differenziert, immer mit Blick auf den jungen Menschen, und verwahrte sich energisch gegen immer härtere und immer neue Sanktionen. Sicher keine leichte Position für eine Justizsenatorin in der damals hoch erregten Debatte. Sehr aufrecht und doch dem Publikum zugeneigt warb sie mit hanseatischer Höflichkeit für eine differenzierte Betrachtung.

Nur wenig später begegnete ich ihr erneut und dieses Mal im kleineren Kreis. Am Rande einer größeren Veranstaltung leitete sie einen Workshop zur Pressearbeit in der Justiz. Eine überschaubare Anzahl von Pressesprecher/inne/n, darunter ich, hatte sich in dem Seminarraum eingefunden und sich zurückhaltend in den hinteren Stuhlreihen verteilt; die vorderen Plätze blieben leer. „Wo sind Sie denn? Kommen Sie nach vorne, hier ist Platz genug. Wenn Sie sich nicht trauen, in der ersten Reihe zu sitzen, werden Sie nicht weit kommen!" So oder ähnlich lautete die energische Begrüßung, auf die ein zögerliches Stühlerücken folgte. Seitdem sitze ich grundsätzlich in der ersten Reihe, wenn sie nicht anderweit reserviert ist.

Ich gratuliere Frau Dr. Peschel-Gutzeit sehr herzlich zu ihrem Geburtstag und wünsche ihr vor allem Gesundheit, damit sie weiterhin engagiert ihrem dritten Beruf nachgehen und Frauen so liebenswürdig wie bestimmt den Weg nach vorne weisen kann. 


\section{Ihre Amtszeit als 1. Vorsitzende des djb (1977-1981)}

\section{Dr. Gisela Wild}

Rechtsanwältin, Hamburg, 2. Vorsitzende des djb von 1977 bis 1979:

Am 10. Juni 1977 wählte der Juristinnenbund (damals „Vereinigung weiblicher Juristen und Volkswirte e.V.“) auf seiner 14. Mitgliederversammlung in Mainz Dr. Lore Maria Peschel-Gutzeit zur Ersten und mich zur Zweiten Vorsitzenden. Das war geplant und vorbereitet. Durch die räumliche Nähe der Arbeits- und Wohnstätten beider Vorsitzenden in Hamburg (Richterin am Hanseatischen Oberlandesgericht die eine, Rechtsanwältin in einer großen Anwaltssozietät die andere) versprachen wir uns eine Steigerung der Effektivität der Verbandsarbeit. Die zu gleicher Zeit beschlossene Neufassung des Vereinszwecks in $\mathbb{S} 1$ Absatz 1 der Satzung von 1977 setzte ehrgeizig erweiterte Ziele. Beschrieb die Gründungsatzung den Zweck des Verbandes noch als „die berufliche und wissenschaftliche Förderung der Mitglieder und die Wahrnehmung ihrer Interessen im öffentlichen Leben “, wurde der Zweck entsprechend dem bereits praktizierten Verständnis auch formal auf das allgemeine Wohl gerichtet. Der Juristinnenbund dient nunmehr laut Satzung dem Zweck, „zur Fortentwicklung des nationalen, supranationalen und internationalen Rechts beizutragen, weltweit die Lage der Frau in Familie, Beruf und Gesellschaft zu verbessern und ihr eine angemessene Partizipation in den verschiedenen Bereichen des beruflichen, politischen und gesellschaftlichen Lebens zu sichern“. Dieser Anspruch, zu allen diesen Bereich betreffenden Fragen gehört zu werden, musste durchgesetzt werden. Die Namensänderung 1979 in „Deutscher Juristinnenbund (Vereinigung der Juristinnen, Volkswirtinnen und Betriebswirtinnen) e.V.“, kurz: „Deutscher Juristinnenbund“ war das Signal nach außen, dem Verband durch qualifizierte Sacharbeit verstärkte Aufmerksamkeit, Anerkennung und Wirkungskraft zu verschaffen. Es ist das Verdienst von Lore Maria PeschelGutzeit, dass das gelungen ist.

Sie und ich hatten uns über das Thema Kinderbetreuung gefunden. Ich führte ein Steuerrechtsverfahren zur Absetzung der Kinderbetreuungskosten als Werbungskosten. Das Finanzgericht Hamburg wies die Klage ab, ebenso der Bundesfinanzhof nach einer surrealen mündlichen Verhandlung. Damit war der Weg zum Bundesverfassungsgericht geebnet. In dem seit 1973 anhängigen Verfahren befragte das Bundesverfassungsgericht auch den Juristinnenbund als Sachverständigen. Dr. Annemarie Mennel und Lore Maria stützten meine Argumente. Diese waren eigentlich unschlagbar: Nach dem Einkommensteuergesetz sind berufsbedingte Kosten absetzbar. Das sind nach dem Gesetz Aufwendungen zur Erwerbung, Sicherung und Erhaltung von Einnahmen. Die berufstätige Frau/ Akademikerin, die eigenes Geld verdient und Steuern zahlt, kann den Beruf nur ausüben und Steuern zahlen, wenn ihr Kind in dieser Zeit betreut wird. Dabei schafft sie zusätzlich einen Arbeitsplatz mit Abgaben an Steuern und Sozialversicherungen. Doch während alle anderen Kosten zur Erwerbung,
Sicherung oder Erhaltung von Einkünften als absetzbare Kosten anerkannt wurden und werden, selbst wenn sie entbehrlich sind (Teppiche, Bilder, Blumen), ordnete das Bundesverfassungsgericht in seiner Entscheidung des Jahres 1977 die für die Berufstätigkeit unabdingbaren Kinderbetreuungskosten dem Bereich der privaten Lebensführung zu und gewährte lediglich den Freibetrag von 1200 DM für außergewöhnliche Belastung auch bei nur einem Kind, nicht erst wie bisher bei zwei Kindern. Die Hausfrau war damit doppelt privilegiert: durch das Ehegattensplitting und durch den Vorteil des unversteuerten Wertes ihrer Arbeitsleistung, der im Rahmen der Zugewinngemeinschaft durchaus anerkannt wurde. Das erforderte auf viele Jahre weiteres Engagement des Deutschen Juristinnenbundes. Erst 2006 erkannte der Gesetzgeber die Absetzbarkeit von Kinderbetreuungskosten bis zu 4000 Euro im Jahr an. Das Thema ist, wie der Streit um das Betreuungsgeld zeigt, noch immer nicht erledigt.

Eine Sternstunde unserer gemeinsamen Amtszeit war die im Herbst 1977 spontan anberaumte Pressekonferenz des Juristinnenbundes in Bonn, in der wir die unter dem Vorsitz von Lore Maria Peschel-Gutzeit von der Familienrechtskommission erarbeiteten Alternativ-Vorschläge zur Neuregelung des ehelichen Sorgerechts vorstellten. Zwei Jahre lang hatten sich die Mitglieder der Kommission - alle Frauen mit Kindern - intensiv mit dem Thema beschäftigt, Lehre und Rechtsprechung kritisch durchforstet, die Regierungsentwürfe von 1973 und 1974 sowie einen dritten aktuellen Fraktionsentwurf geprüft und kräftig miteinander diskutiert. Die Kommission legte einen vollständigen, durchredigierten Gesetzestext mit Begründung vor, einschließlich Begleitgesetzen. Er lieferte neue Gedanken, die sich von den bisherigen Vorschlägen vielfach deutlich unterschieden: Sie waren allein am Wohl des Kindes ausgerichtet. Nach Billigung durch die Mitgliederversammlung 1977 in Mainz wurde der Text als Buch gedruckt, um größere Resonanz zu gewinnen. Zunächst vergebens: Der Antrag des Juristinnenbundes, mit seinem Alternativentwurf vom Bundestag gehört zu werden, wurde abgelehnt. Begründung: Es könnten keine weiteren Sachverständigen geladen werden. Unter den zwölf geladenen Sachverständigen befand sich keine Frau.

Also gingen Lore Maria und ich wenige Tage vor der Sachverständigenanhörung im Deutschen Bundestag mit der besagten Pressekonferenz in Bonn an die Öffentlichkeit. Das Echo war gewaltig. Die vom Juristinnenbund zur Reform des elterlichen Sorgerechts entwickelten Gedanken in den Einzelfragen, die wir vortrugen, fanden an dem Tag nur geringe Beachtung - die differenzierten Feinheiten wurden von den meisten Journalisten gar nicht so schnell erfasst. Dafür zündete schon bald nach Beginn der Pressekonferenz die im alternativen Gesetzentwurf vorgesehene Abschaffung des Züchtigungsrechts. Das vom Juristinnenbund geforderte generelle Verbot von Gewalt innerhalb der Familie erhitzte die Gemüter im Saal bis hin zu tumultartigen Szenen. Da half 
es nicht, dass wir auf die inzwischen längst vollzogene Abschaffung körperlicher Züchtigung im Militär, beim Gesinde, in den Schulen und gegenüber der Ehefrau (1928) verwiesen, die Erregung ob der ungeheuerlichen Forderung eskalierte derart, dass wir zeitweise fürchteten, selbst Prügel zu beziehen. Tags darauf waren die Medien voller Berichte über den Auftritt und damit auch über den alternativen Gesetzentwurf des Juristinnenbundes.

Wenige Tage später erhielt Lore Maria die Einladung, als Sachverständige an der bevorstehenden Anhörung vor dem
Rechtsausschuss des Deutschen Bundestags teilzunehmen. Fast zwei Jahre warben wir weiter für unsere Alternativen. Am 18. Juni 1979 wurde das Gesetz zur Neuregelung des Rechts der elterlichen Sorge vom Bundestag verabschiedet. Wesentliche Vorschläge des Alternativentwurfs waren darin übernommen, teils wörtlich, teils dem Sinn nach. So auch das Züchtigungsverbot.

Noch vieles mehr gäbe es zu berichten, was Lore Maria Peschel-Gutzeit zu danken ist. Ihr feminines Netzwerk hält bis heute, und das tut gut.

\section{Der djb gratuliert}

\section{Dr. Ulrike Bick}

zum Amtsantritt als Richterin am Bundesverwaltungsgericht am 10. August 2012. Dr. Ulrike Bick, geb.1959 in Herne, studierte in Bochum, Genf und Paris Rechtswissenschaft. Nach Promotion durch die Ruhr-Universität Bochum 1988 und Zweitem Staatsexamen begann sie 1992 ihre richterliche Laufbahn beim Verwaltungsgericht Gelsenkirchen. Nach einer zweieinhalbjährigen Abordnung an das Bundesverwaltungsgericht (2000-2002) wurde sie im Mai 2002 zur Richterin am Oberverwaltungsgericht für das Land Nordrhein-Westfalen ernannt. Von 2006 bis 2009 war sie als wissenschaftliche Mitarbeiterin an das Bundesverfassungsgericht abgeordnet. 2011 wurde sie Vorsitzende Richterin am Oberverwaltungsgericht für das Land Nordrhein-Westfalen. Dort übernahm sie die Leitung des 16. Senats. Das Präsidium des Bundesverwaltungsgerichts hat ihr nun den 9. Revisionssenat zugewiesen, der u.a. für Straßen- und Wegerecht, Kommunalabgabenrecht und Flurbereinigungsrecht zuständig ist. Dr. Bick ist seit 1996 Mitglied des djb.

\section{Petra Heinicke}

Rechtsanwältin, München, zur Verleihung des Bundesverdienstkreuzes am Band am 2. Oktober 2012 aus der Hand des Bayerischen Staatsministers für Wissenschaft und Kunst, Dr. Wolfgang Heubisch. Petra Heinicke ist dem djb 29-jährig im Jahr 1988 beigetreten. Sie war von 1991 bis 1997 Vorsitzende der Regionalgruppe München/Südbayern im djb und gehörte dem djb-Bundesvorstand von 1993 bis 1995 als Beisitzerin an. Seit 1999 ist sie Vorsitzende des Münchener Anwaltvereins und jetzt auch DAV-Vorstandsmitglied. Darüber hinaus gehört sie dem Vorstand der RAK München und der Satzungsversammlung der BRAK an.

\section{Gudrun Schattschneider}

zu Übernahme der Leitung des neuen Hauptstadtbüros des Arbeiter-Samariter-Bundes (ASB) am 1. September 2012. Gudrun Schattschneider, geb. 1969, ist als Rechtsanwältin zugelassen. Sie war zuvor stellvertretende Leiterin der Bundesgeschäftsstelle des Deutschen Bundeswehrverbandes (DBwV) in Berlin. Sie leitete dort außerdem das Ressort für Grundsatzfragen und Recht. Seit 1993 ist sie Mitglied des djb.

\section{Dr. Christina Stresemann}

zur Ernennung als Vorsitzende Richterin am Bundesgerichtshof durch den Bundespräsidenten am 3. September 2012. Dr. Christina Stresemann, geb. 1957 in Berlin, studierte an der Freien Universität Berlin (FU) Jura, wo sie nach Abschluss ihrer Ausbildung zunächst als Wissenschaftliche Mitarbeiterin blieb. 1987 trat sie in den höheren Justizdienst des Landes Berlin ein. 1988 wurde sie an die Berliner Senatsverwaltung für Justiz und Bundesangelegenheiten abgeordnet, wo sie u.a. die Aufgaben einer persönlichen Referentin der Justizsenatorin Prof. Dr. Jutta Limbach wahrnahm. 1990 wurde sie zur Richterin am Landgericht Berlin ernannt, wohin sie 1992 zurückkehrte. 1995 wurde sie für drei Jahre als Wissenschaftliche Mitarbeiterin an das Bundesverfassungsgericht abgeordnet und außerdem zur Richterin am Kammergericht befördert. 2003 wurde sie zur Richterin am Bundesgerichtshof ernannt und dem V. Zivilsenat zugewiesen, der insbesondere für das Grundstücks-, Nachbar- und Landpachtrecht zuständig ist. Seit 2007 gehört sie dem Präsidium und seit 2010 dem Präsidialrat des Bundesgerichtshofs an. Seit 2009 nimmt sie zudem die Aufgaben der Beauftragten für Auslandskontakte wahr.

Von 2004 bis 2012 war sie Richterin des Verfassungsgerichtshofs des Landes Berlin. Dr. Christina Stresemann ist seit 1999 Mitglied des djb.

\section{Regina Vieler}

zur Ernennung als ehrenamtliche Beisitzerin im Senat für Wirtschaftsprüfersachen des Bundesgerichtshofs zum 1. September 2012. Von den zehn Senatsmitgliedern ist sie die einzige Frau.

Regina Vieler, geb. 1957 in Lübeck, studierte von 1976 bis 1981 Betriebswirtschaftslehre an der Technischen Universität Berlin. Nach ihrer Diplomprüfung 1981 und den Prüfungen zur Wirtschaftsprüferin 1987 und Steuerberaterin 1986 befasste sie sich vor allem mit der Prüfung und der steuerlichen Beratung von Unternehmen jeder Größe und aus allen Branchen sowie der Prüfung und Beratung auch von DAX-Unter- 